\title{
What an exciting time it is: a pictorial essay of Doppler sonography
}

\author{
Shoichi Matsutani ${ }^{1}$
}

(C) The Japan Society of Ultrasonics in Medicine 2016

There are numerous blood vessels in the human body, and the vascular system has an essential role to maintain our vital life. However, some blood vessels also play an essential role in the pathophysiology of diseases. Among the human blood vessels, the coronary artery is considered to be the most famous one in such contexts because pathological conditions involving this artery cause very serious heart disease called coronary heart disease or ischemic heart disease in human beings around the world. However, the human body has another blood vessel with a circular course like the coronary artery, i.e., the coronary vein. Cardiologists may not show any interest in this blood vessel, because it is not a vein in the heart. The coronary vein belongs to the venous system of the stomach. Most gastroenterologists also do not have any special interest in this vein in their daily clinical practice, because the coronary vein does not have any pathological significance in common with stomach diseases. However, this vein takes on clinical significance when portal vein pressure begins to increase and results in portal hypertension. The reason why I focused on this vein in this month's editorial is because my research spanning almost 40 years has been deeply connected to the clinical aspects of this disease as they relate to sonographic investigations. I may have to thank some readers for their kind consideration if they feel the content of this month's editorial is too personal.

Among the venous system of the stomach, two major gastric veins, the left gastric vein and the right gastric vein, join at the lesser curvature of the stomach. This

Shoichi Matsutani

matsu803@pg7.so-net.ne.jp

1 Department of Gastroenterology, Funabashi Municipal Medical Center, Chiba, Japan communication makes a kind of loop between two sites where the two veins separately enter into the portal vein (Fig. 1). This shape is thought to be the reason why this vein is called the coronary vein. Of these two components of the coronary vein, the left gastric vein has another important communication with the esophageal vein at the cardiac portion of the stomach (Fig. 1). This connection is crucial because the coronary vein poses a potential risk in portal hypertension by reversing the flow direction and consequently forming esophageal varices, which frequently cause massive and fatal hemorrhage (Fig. 2).

Nowadays, we are able to demonstrate the coronary vein (the left gastric vein: LGV) using high-resolution ultrasonography even in normal conditions in daily practice (Fig. 3). However, it was a bit different in the late 1970s when I started sonographic investigations of the coronary vein in portal hypertension because real-time gray-scale ultrasonography and pulsed-Doppler ultrasound were still under development (Fig. 4). The initial aim of my investigations was to support non-surgical treatments of esophageal varices using interventional radiology or endoscopy, which were rapidly developing in the late 1970s. Because of the limited length of this short editorial, I would like to show some representative sonographic images of the coronary vein from my investigations in place of presenting the detailed results of my study (Figs. 3, 4, 5). I would be so delighted if the readers could just enjoy these images without considering the complicated pathophysiology of portal hypertension. Based on my studies, I can confidently say that ultrasonography is the best tool for investigating the hemodynamics of small vessels like the coronary vein in real time, which other imaging modalities can not easily approach. I have had several exciting moments when I observed very rapid changes in Doppler waveform after vasoactive stimulations 


\section{Lt. gastric $v$.}

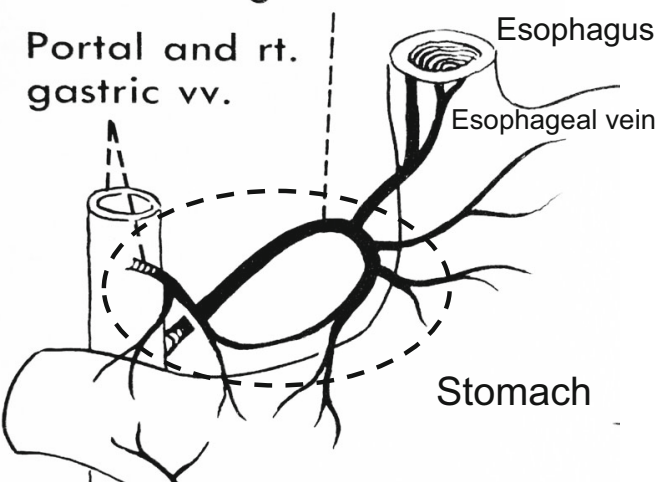

Fig. 1 Anatomical view of the coronary vein

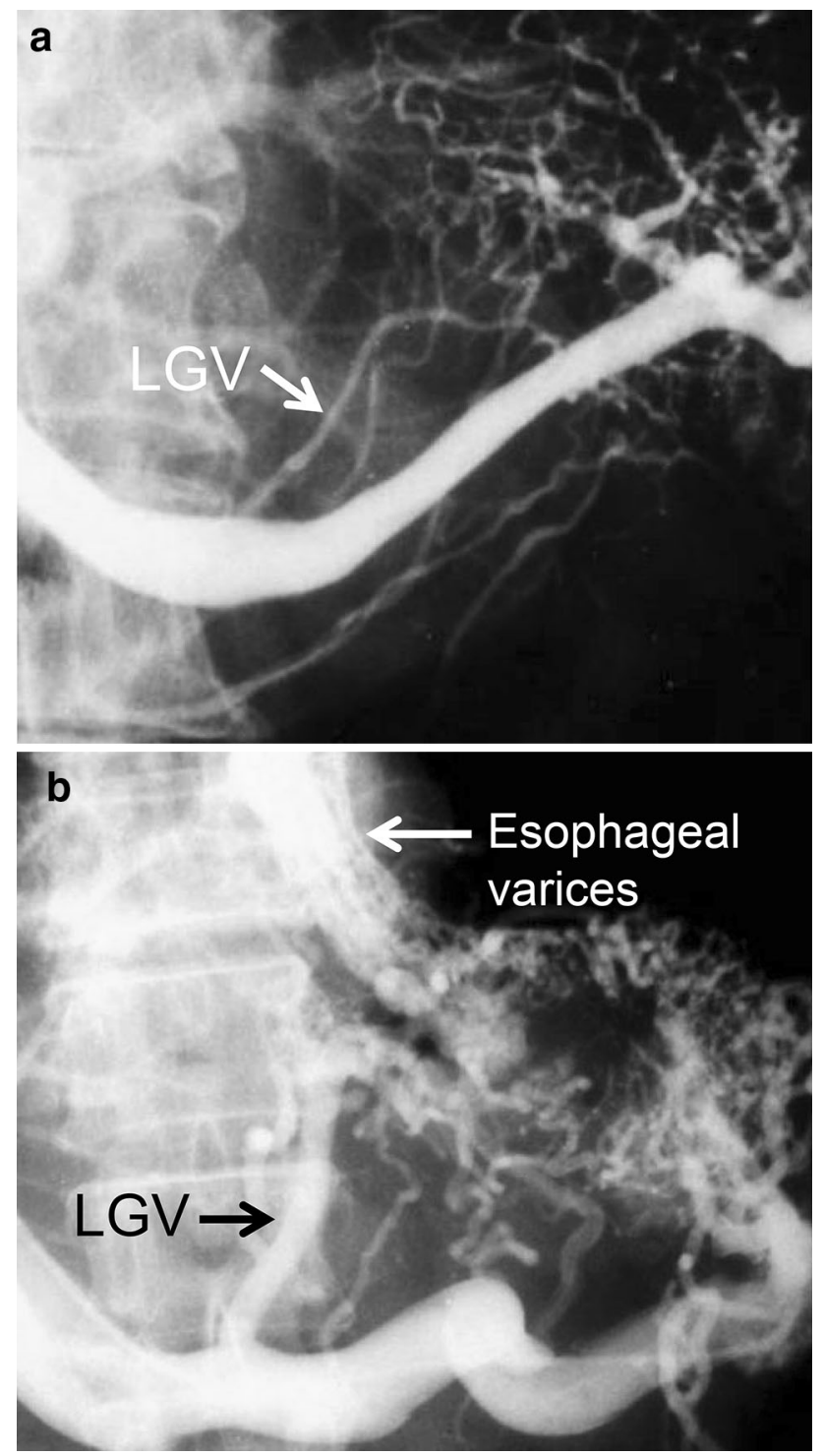

Fig. 2 Radiographic view of the coronary vein. a The normal left gastric vein. b The left gastric vein and esophageal varices in portal hypertension
(Fig. 5). Every reader may have a similar feeling at least once during his or her career in specific fields of clinical practice or research with ultrasonography.
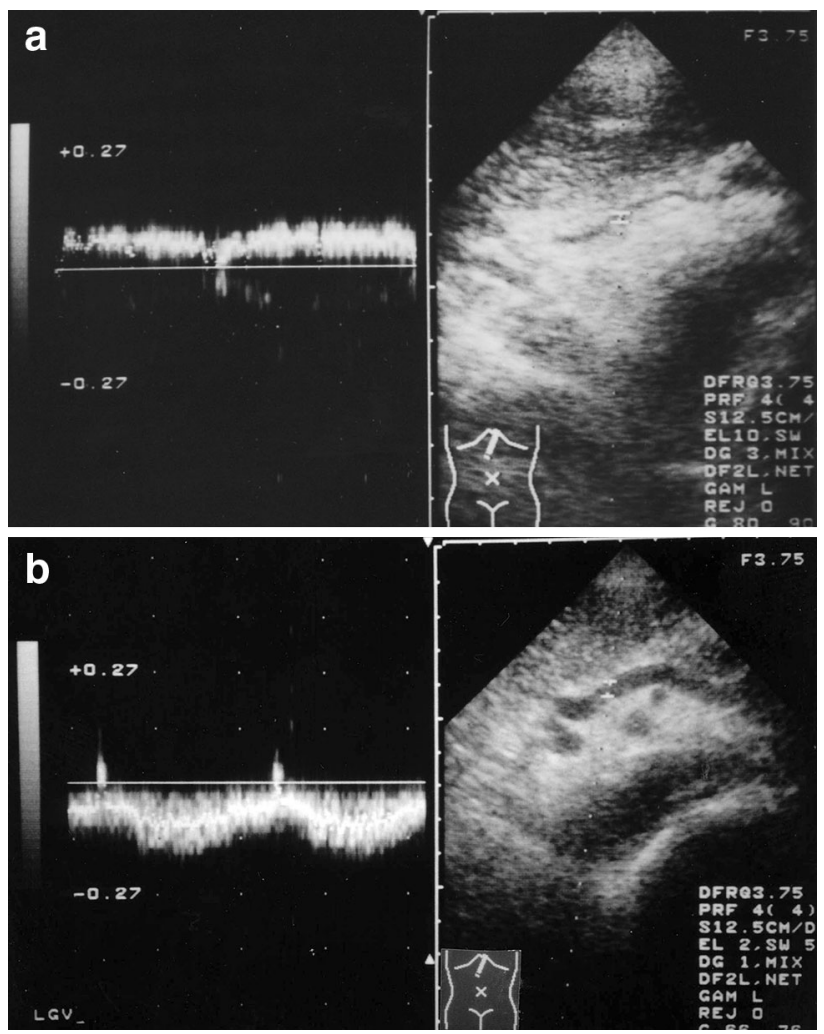

Fig. 3 Ultrasonographic view of the coronary vein. a The normal left gastric vein with flow direction to the liver (forward flow or hepatopetal flow). b The left gastric vein in portal hypertension with flow direction to esophageal varices (reversed flow or hepatofugal flow)

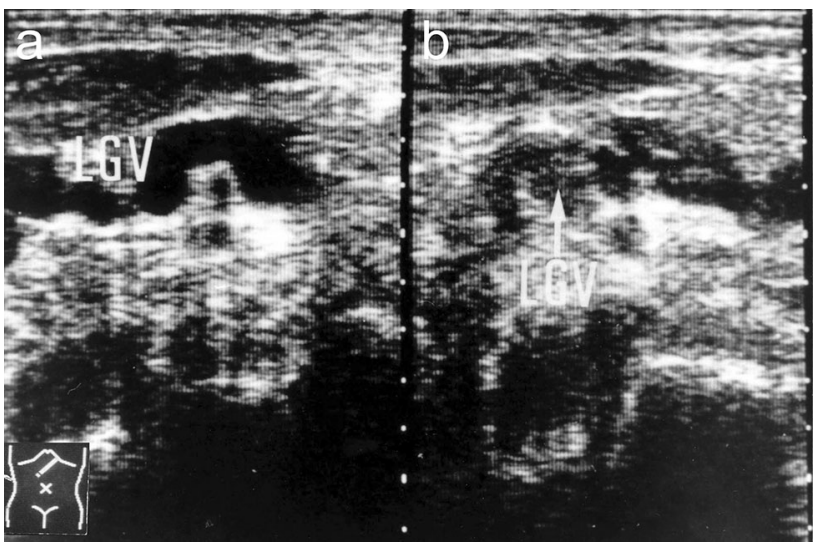

Fig. 4 Sonographic identification of the coronary vein using contrast enhancement with direct injection of radiological contrast medium with physiologic saline through a catheter (from a study in the late 1970s). a Before injection of saline into the coronary vein. b Contrast enhancement in the coronary vein during radiological contrast medium with saline injection 


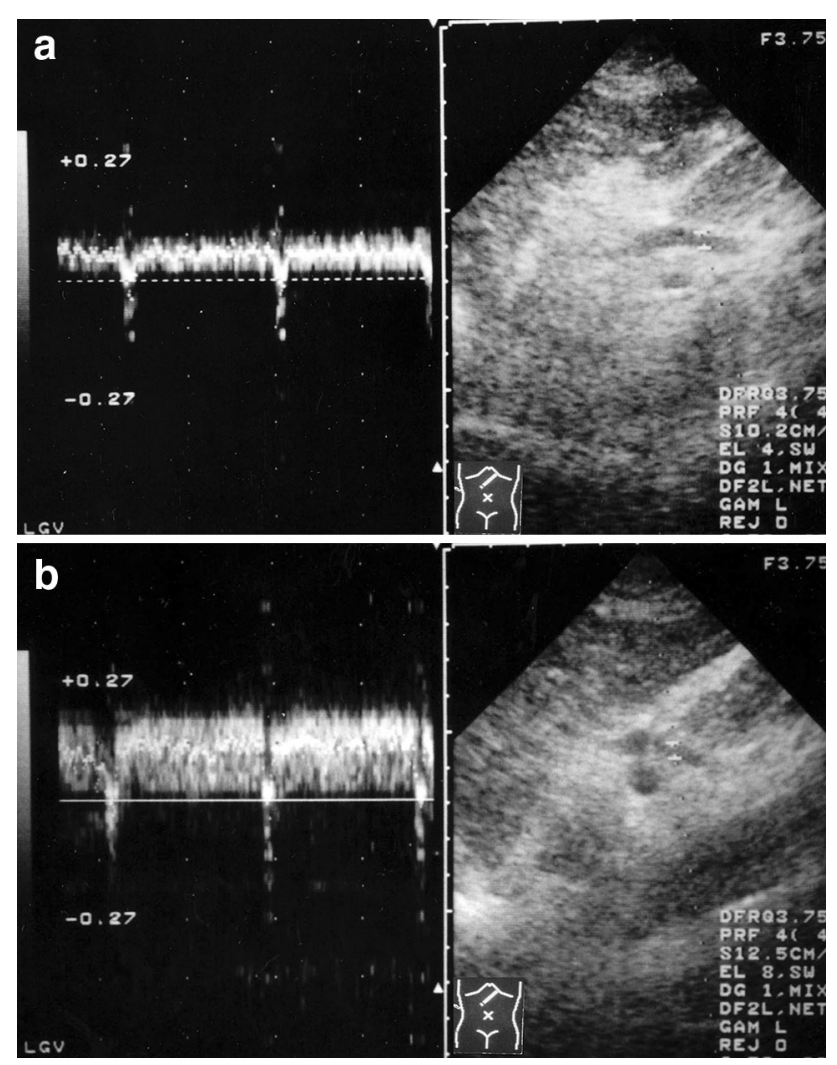

hemodynamic analysis with ultrasonography. The editor strongly believes that ultrasonographic investigation will be the royal road to elucidating the hemodynamic nature of diseases in the past, the present, and the future.

Fig. 5 Doppler waveform changes in the coronary vein with oral glucose intake (from the first case in my study in the late 1980s). a Before oral glucose intake. b After oral glucose intake

Even now, there must be many disease-related blood vessels in the human body waiting for further 